\title{
Utilization of Deep Learning Models in Medical Industries
}

\author{
N. Thirupathi Rao ${ }^{1}$, Debnath Bhattacharyya ${ }^{2 *}$ \\ ${ }^{1,2}$ Department of Computer Science and Engineering \\ Vignan's Institute of Information Technology \\ Visakhapatnam, AP, India, \\ ${ }^{1}$ nakkathiru@gmail.com, ${ }^{2}$ debnathb@gmail.com
}

\begin{abstract}
Deep learning is the rapidly growing technology in the field of almost all recent technologies in the market. With the successful application of deep learning in almost all the recent technologies, the growth in all the areas was splendid. The growth in those areas has crossed the expectations of the scientists. Recently, the application of deep learning in the areas of medicine and pharma is growing in recent times in a rapid fast. In the current paper, the authors represent the seven applications or areas in the field of medicine and pharma where the applications of deep learning were implementing and good results are obtaining.
\end{abstract}

Keywords: Deep learning, medicine, pharma, supervised learning, unsupervised learning, artificial intelligence, artificial neural networks.

\section{Introduction}

Deep learning area is the recent technology or the recent strategies that were being implemented in the areas of health and pharma such that to get good results. Both the areas are the never ending or the never stopping research areas. Always the research in these both areas has to be run continuously such that to identify good solutions to the available diseases in the society. The research on these areas was always a never ending process such that to find the new set of drugs or the new set of solutions to the never ending and new set of diseases occurring in the society. Deep learning is the effective technology that can be used in the field of medicine and pharma. By the utilization of these deep learning techniques in those two areas, the results we can achieve may be very good and very attractive and encouraging results. Some of the scientists are expecting and estimating a huge amount of revenue might generate in the area of applying the field of deep learning to the areas of the medical and its related areas [1].

A scientist known as McKinsey had estimated the amount that can be spent or revenue may be generated by utilizing these techniques in the field of medicine or the pharma may reach the value of $\$ 150 \mathrm{~B}$ by every year. It is the revenue that can be generated by combining the areas of both deep learning and Bigdata. It can be achieved by utilizing various new techniques like decision making, innovation working with optimized values, efficiency to be improved and clinical or the research trials. Some of the other techniques or the devices that can support these areas are the devices that can be helpful for the doctors or the patients are a tool kit that can be helpful for the physician, consumers [2]. Some of the other tools also helpful to the set of people like the insurers and the regulators to handle the health related issues.

Article history:

Received (May 9, 2019), Review Result (September 23, 2019), Accepted (October 28, 2019) 
In order to conduct such a large and huge research such that to develop and design the devices for health related issues, developers need a huge data for processing and also to study. In order to study the performance of the developed models or designs, huge data was required. This data was collected from various doctors, physicians, from the various set of records that were variable in the various set of hospitals around the world. The data and symptoms may change from time to time and the place to place [3]. It is due to various local conditions, temperature conditions and other geographical related issues. This information is much useful in establishing the good health infrastructure and good research setup data for future research of these areas.

In order to meet the day to day needs and to meet the day to day research requirements, the synchronization of data, analysis of data and processing of such data was always required. The deep learning and its related techniques can be used and processed for the research areas like pharma and medicine. The cases or the applications where the deep learning and its related areas can be utilized for further processing and to conduct research was discussed in the following sections. Seven applications where this deep learning can be used was discussed as follows,

The areas of Medicine and Pharma where Deep learning can be used are,

Disease diagnosis or identification of disease.

Discovery of drugs.

Prediction of Diseases

Research on clinical trials

Radiology and Radiotherapy

\section{DISEASE DIAGNOSIS OR IDENTIFICATION OF DISEASE}

It is a saying from all the people that prevention of disease is more important than curing of the disease. In the similar fashion, the identification of diseases is more famous and important such that to provide the correct treatment and provide the correct treatment in the proper important time. Identification of the disease in the proper time is more important for proper utilization and proper provision of the correct treatment for the people. Hence, due to the provision of the correct treatment at the right time can reduce the problem of seriousness in the diseases and the life of the persons can be saved by the same logic. Hence, the important point is to identify the disease or to predict the disease such that to identify the disease at the right time and to provide the right medicines or treatment to the exact disease at good or right time. The main motto of going for the deep learning research was to identify the disease and the related issues for choosing of the deep learning research in medicine[4].

As per reports issued by an association in the United States which was working for the pharma research and clinical research was about 800 medicines were being under trial for cancer treatment. In such cases, by using the deep learning techniques, we can reduce the time taking for checking the vaccines or the medicines for cancer treatment. Major companies or industries or the laboratories were working particularly for the identification of diseases and also for the treatment of the diseases was cancer. The most important area of research going on in the major companies for identification of the cure for the cancer disease and large companies were behind the treatment of the same disease. In the year 2016, the IBM Watson health bulletin released on the research area likes Genomics. This company was had a partnership with Quest Diagnostics, which was targeted to provide précised medicine for the genome tumor or tumors in the human brain[5]. 
Another big organization known as Berg, which was located at Boston was working on the area of artificial intelligence research such that to develop and find the diagnostics processes to various diseases and the therapeutic treatments in various areas of medicine as oncology, radiology, etc. The other research areas and the other experiments were going on was on tumor detection and the identification of the correct dosage of medicine under various trials and also the research areas was going on the other disease called the cancer. The detection of the tumor in the human brain as most important because, the presence of the tumor may give more problems to the people without the consideration of the age of the patients. The tumor forming and growth of the tumor in the human brain was independent of the human age and the human gender. It may be observed in any age of persons and also in any gender of human beings. The other important areas of research going on in the field of medical research with the usage of deep learning techniques was the Google's DeepMind Health applications, which was announced and working in the country of UK such that to identify solutions to the problems of eye problems [6].

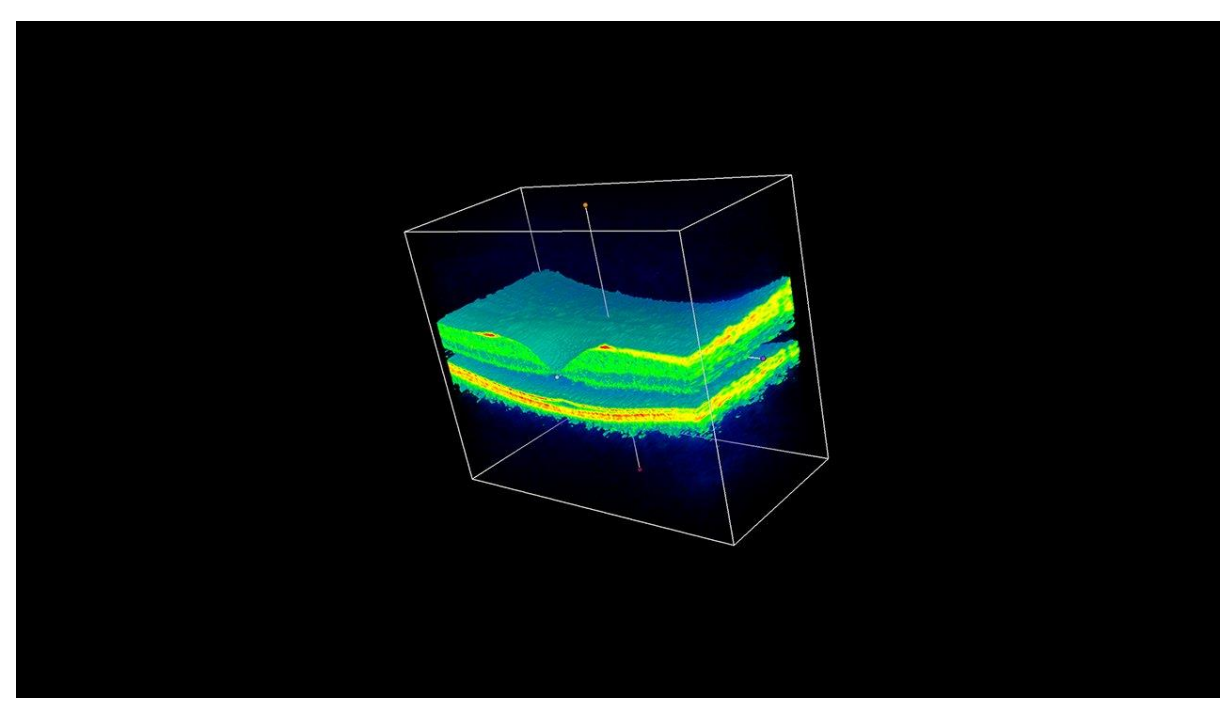

Figure. 1 Google DeepMind Health - An OCT scan of one of the DeepMind Health team's eyes

\section{THE DISCOVERY OF DRUGS}

The utility of the deep learning techniques in the preliminary stages or the duration of drug tests was the most recent applications or the trend going in the field of medical and artificial intelligence areas. The usage of deep learning techniques in drug discovery or the trials of the drug utility may have the good impact on predicting the performance behaviour of drugs without huge loss or less damage to the persons for checking. The success rate on the usage of deep learning techniques in the drug discovery or the prediction the drug behaviour was good and also it reflects the screening of drug compounds. The success rate was so good and at the high rate compared to other techniques or models. The next generation technology to be used for the prediction of the genetic sequencing was implemented in the current models.

Providing the précised medicine for the disease and identification for the précised medicine for the disease was the major task to be considered here. It is also required to identify various available alternative medicines and various therapies for identification and usage of the medicines to diseases. It is also required to identify the alternative paths and ways to identify 
and observe the diseases and therapies for those identified diseases. The major technique utilized was the unsupervised learning technique such that to identify the disease and also to predict the précised medicine for the disease. It is also used to identify the large patterns of the data in the pattern of the data that was available for existing diseases [7].

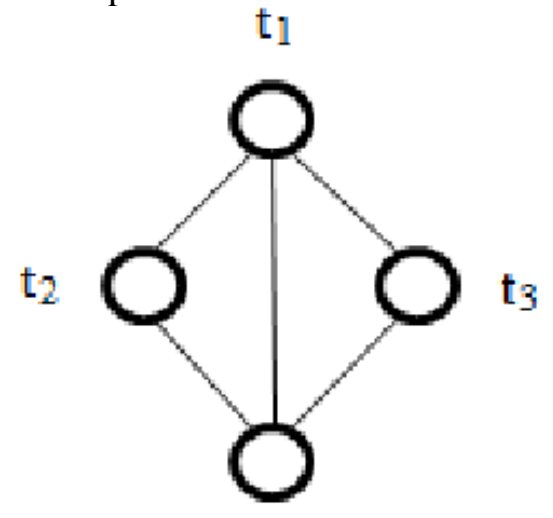

\begin{tabular}{c|c|c|c|c|}
\multicolumn{1}{c}{} & \multicolumn{1}{c}{$\mathrm{t}_{1}$} & \multicolumn{1}{c}{$\mathrm{t}_{2}$} & \multicolumn{1}{c}{$\mathrm{t}_{3}$} & \multicolumn{1}{c}{$\mathrm{t}_{4}$} \\
\cline { 2 - 5 } $\mathrm{r}_{1}$ & 1 & 0 & 0 & 1 \\
\cline { 2 - 5 } $\mathrm{r}_{2}$ & 0 & 1 & 0 & 0 \\
\cline { 2 - 5 } $\mathrm{r}_{3}$ & 0 & 0 & 1 & 0 \\
\cline { 2 - 5 } & & &
\end{tabular}

Initial Position Matrix

\section{Sample Workflow}

\begin{tabular}{|c|c|c|c|}
\hline $\mathrm{t}_{1}$ & $\mathrm{t}_{2}$ & $\mathrm{t}_{3}$ & $\mathrm{t}_{4}$ \\
\hline 4 & -1 & 2 & 1 \\
\hline-2 & 3 & -4 & -3 \\
\hline 0 & 2 & 5 & -1 \\
\hline
\end{tabular}

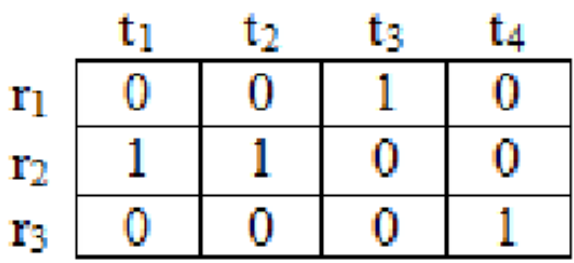

Initial Velocity Matrix

\begin{tabular}{l|c|c|c|c|}
\multicolumn{1}{c}{} & \multicolumn{1}{c}{$\mathrm{t}_{1}$} & \multicolumn{1}{c}{$\mathrm{t}_{2}$} & \multicolumn{1}{c}{$\mathrm{t}_{3}$} & \multicolumn{1}{c}{$\mathrm{t}_{4}$} \\
\cline { 2 - 5 } $\mathrm{r}_{1}$ & 2 & -1 & 4 & -1 \\
\cline { 2 - 5 } $\mathrm{r}_{2}$ & 0 & 3 & -4 & -3 \\
\cline { 2 - 5 } $\mathrm{r}_{3}$ & 0 & 2 & 3 & 1 \\
\cline { 2 - 5 } & & & &
\end{tabular}

Updated Velocity Matrix

\section{Initial pbest Matrix}

\begin{tabular}{c|c|c|c|c|}
\multicolumn{1}{c}{} & \multicolumn{1}{c}{$\mathrm{t}_{1}$} & $\mathrm{t}_{2}$ & $\mathrm{t}_{3}$ & \multicolumn{1}{c}{$\mathrm{t}_{4}$} \\
\cline { 2 - 5 } $\mathrm{r}_{1}$ & 1 & 0 & 1 & 0 \\
\cline { 2 - 5 } $\mathrm{r}_{2}$ & 0 & 1 & 0 & 0 \\
\cline { 2 - 5 } $\mathrm{r}_{3}$ & 0 & 0 & 0 & 1 \\
\cline { 2 - 5 } & & & &
\end{tabular}

Updated Position Matrix

Figure. 2 Sample Matrix model [11]

From the above image, it is observed that it can be used to identify or to utilize four sets of data or four sets of things to identify the various ways of diseases or the cure for those diseases. The A matrix will provide us the representation of both supervised and unsupervised learning problems. The patient data was represented and analyzed for identifying the disease symptoms. The B image is used for representing the various features of the diseases such that to identify and the disease symptoms can be represented in the form of the decision trees. The third image used in the above image as the neural network model. This neural network model can be used for identifying and the prediction of outcomes which were based on the features of the diseases. Hence, this neural network model can be used for the transformation of these symptoms such 
that to identify the diseases based on the symptoms. The fourth and the last part of the D is used for representing the k-nearest neighbour model such that for providing the training classes for the required problems and also used for training purposes in the similar cases of the diseases [8].

The major research conducting units or the universities which were mostly working on the identification of diseases and also used to identify the predicting the diseases and their curing processes or the method was the MIT Clinical deep learning Group. The major research conducting by this laboratory was to identify the disease symptoms and tries to identify the best fit algorithms and other effective treatment of those diseases. The major research of this laboratory was to understand and identify the disease symptoms and gene processing and effective treatment processes for the diseases like the diabetes and Type 2 diabetes.

The optimization of the utilization of the medicines and also the manufacturing of the medicines will be processed and utilized by the UK Royal Society. This society is using the deep learning techniques such that to implement in the areas of bioinformatics. The research on deep learning and its related techniques by this society was very much helpful to the manufacturers of pharmaceuticals and other related areas of medical diseases.

\section{THE PREDICTION OF DISEASES}

The utilization of deep learning and its related techniques in the field of medical and its related areas was becoming popular day by day. As the prediction of symptoms from various disease changes from time to time and also change due to various factors like the temperature, the area and other tropical conditions. Hence, in order to predict the diseases or to identify the exact diseases, we need to implement some good and sound artificial intelligent technique or some good deep learning technique such that to predict the disease exactly or with some good matching of the symptoms. These techniques are used for identifying the disease around the world. These systems or the techniques used in systems will use the data that was collected from various sources like satellites and historical data to identify the diseases. These systems may also collect data from other sources from web, social media and other supporting sources of data. For identifying the serious diseases like malaria, the deep learning techniques that were used mostly are support vector machines and artificial neural networks. By using these two techniques, the outburst of the malaria diseases was happened. The various symptoms that were considered for identifying the malaria disease are body temperature of the patient, rainfall in average value on the area of the patient, the number of positive cases in the area and other points [9].

The out breaking of various severe diseases is taking time in some countries and in some countries it is so fast to outburst. Especially the out bursting of the patient diseases is very high in the recent developing countries with the huge number of people in these countries. This situation is happening in the countries with less medical infrastructure, less education to the people in the country and lack of major treatment facilities to the common people in those countries.

\section{RESEARCH ON CLINICAL TRIALS}

The usage of deep learning and its related techniques in the areas of research on clinical trials is the recent trends in recent years. The applications that were available for clinical trials were having very huge and high advantages compared to the other set of clinical trial methods. By applying this sort of advanced techniques such that to identify and they predict the behaviour of various medicines and various symptoms of diseases. By using these techniques, the cost of 
clinical trials can be reduced more. By this technique, the loss of the human lives for clinical trials can also be reduced in good number. The data that was available can also be used for doctors such that to take good decision on the various symptoms available in the society. These techniques are much useful in those cases of genetic models to be testing and analyzing the behaviour of these sorts of techniques. The most benefits of using these techniques will provide us the results very fast, secure and less damage to the human lives and the cost of experimentation can be reduced to some good amount. By using these techniques of deep learning, the trials of various medicines or drugs can be completed with a very less amount of money.

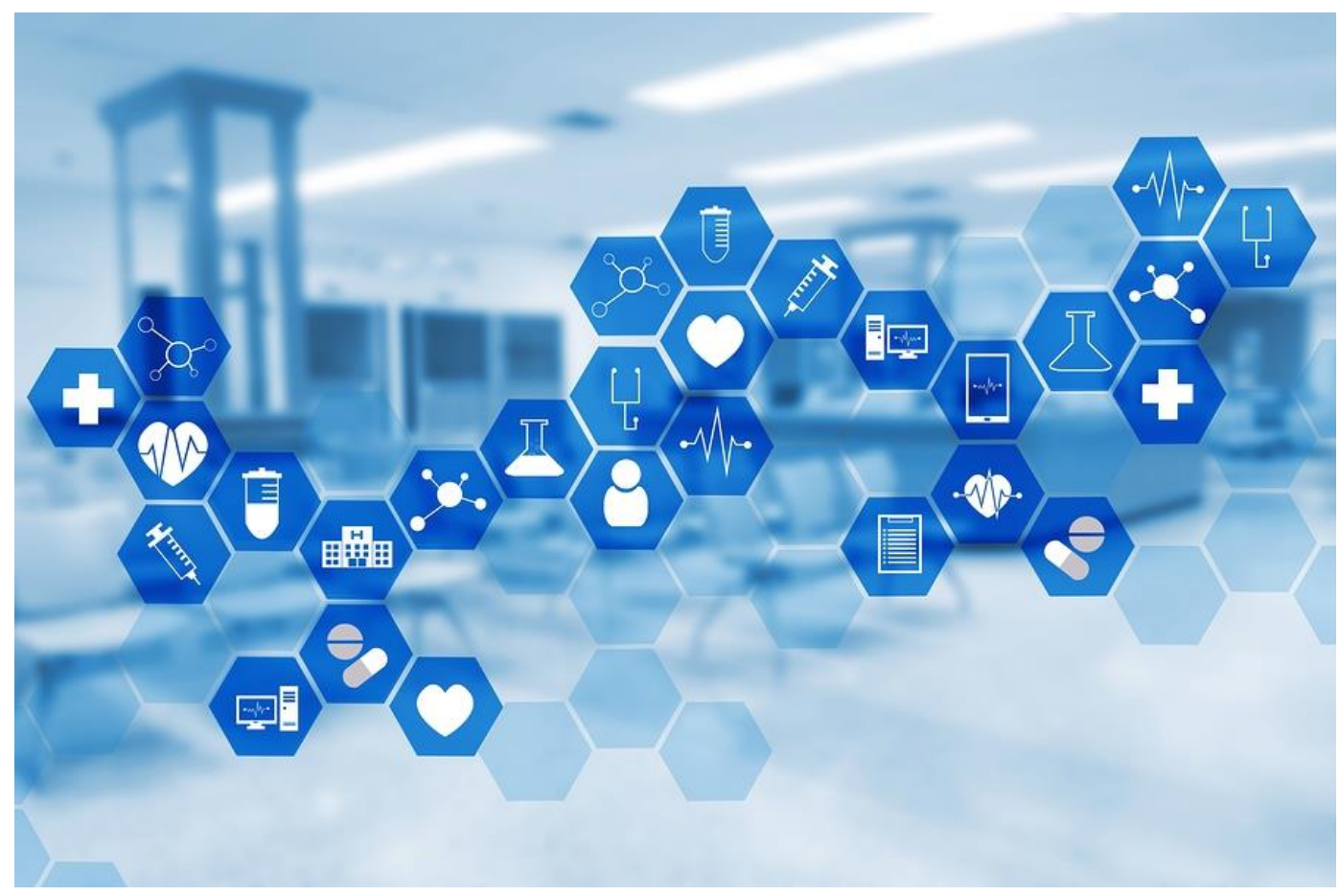

Figure. 3 Clinical Trials Cost estimation [12]

The deep learning techniques can also be used in various machines which were working in remote areas where human beings cannot go to such places and also collect the data and process that particular data for identification of diseases and other related issues of those problems. These techniques can be used for monitoring the real time datasets and real time data access for increased safety. The data may be from biological data monitoring which can be more dangerous to human beings. Further, these techniques also provides us to make the data in the form of electronic entries such that to reduce the duplicate of data or the erasing fi the data related to disease and other related issues of that particular data [10].

\section{RADIOLOGY AND RADIOTHERAPY}

The identification of cancer causing tissues in the human body was most important. If these tissues were identified in good time with good stage of the cancer causing tissues, the disease can be cured very easily with various solutions or the treatments available in the medical areas today. Various reports from various patients from all over the world are made as records and sued for identifying the major symptoms that were causing the cancer diseases. Using the 
supervised learning techniques in the deep learning models was famous all over the world such that to identify the symptoms that were available in the hospitals available today to identify the cancer diseases. Several related applications were developed and testing from day to day and time to time such that to match the available symptoms to finalize or identify the cancer diseases.

It is also important to identify the stages that were available in the cancer diseases. The severity of the disease can be taken based on the stages of the disease. The range of the stages increasing from first stage to the fifth stage, the severity of the diseases increases seriously. As the severity of the disease increases, the death of the patient also takes place in various cases. Hence, the identification of the stage of the cancer tissue sin the body is more important for the predicting machines or the predicting systems to save the human lives. As the symptoms from each stage of the disease varies, it is very important very difficult to identify the stage and also the challenging issue.

The major companies or the research laboratories working on the cancer identification are Google DeepMind Health radiotherapy planning model. Now, the Google was working on this technology and trying to identify the solutions for the diseases and also to provide the range of radio therapy we need to provide such that to reduce the cancer tissues and also not affect the human body other parts. By using the supervised learning methods and artificial neural network models such $\mathrm{h}$ that to identify the cancer tissues and the stages of the cancer disease, proper treatment at proper timing can be done. The following figure gives the radiotherapy images of the person who is undergoing the therapy.

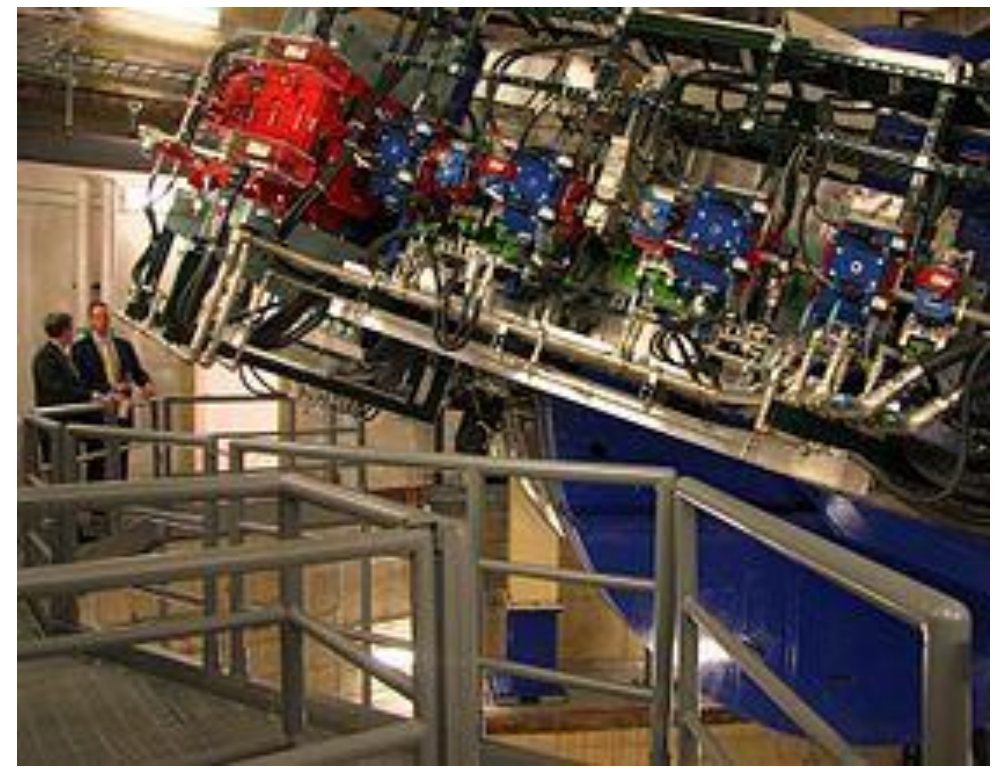

Figure. 4 Google DeepMind Health - radiotherapy planning [13]

\section{CONCLUSIONS}

In the current paper, the details about the deep learning techniques and the utility of the deep learning techniques in the areas of pharma and medicine was discussed in detail. The seven factors where the deep learning and artificial intelligent techniques can be used for resolving and finding the solutions were discussed in detail. The detailed analysis about the various issues to be considered for using these techniques in disease, identification, symptom analysis, cancer disease identification and the suggestion of radiotherapy was discussed in detail. 


\section{References}

[1] https://www.techemergence.com/machine-learning-in-pharma-medicine/ [Last accessed on 12-10-(2018)].

[2] https://www.igeahub.com/2018/08/28/evaluation-of-clinical-trial-costs-and-barriers-to-drug-development/ [Last accessed on 12-10-(2018)].

[3] Gulshan, V., Peng, L., Coram, M., Stumpe, M. C., Wu, D., Narayanaswamy, A., et al. "Development and Validation of a Deep Learning Algorithm for Detection of Diabetic Retinopathy in Retinal Fundus Photographs". Jama, Vol.316, No.22, pp.2402-2405. (2016) doi:10.1001/jama.2016.17216

[4] Esteva, A., Kuprel, B., Novoa, R. A., Ko, J., Swetter, S. M., Blau, H. M., \& Thrun, S. (2017). "Dermatologistlevel classification of skin cancer with deep neural networks. Nature", Vol.542, pp.115-118.DOI: 10.1038 /nature21056

[5] Salvador Garc'1a, Juli' an Luengo, and Francisco Herrera. "Data pre-processing in data mining". Springer, (2015). DOI: 10.1007/978-3-319-10247-4

[6] Veronica Bol’on-Canedo, Noelia S’anchez-Marõno, and Amparo Alonso-Betanzos. "Feature Selection for High-Dimensional Data". Springer, (2015).

[7] Emilie Renard, Andrew E. Teschendorff, and Pierre-Antoine Absil. "Spatiotemporal ICA improves the selection of differentially expressed genes". In European Symposium on Artificial Neural Networks, Computational Intelligence and Deep learning, (2016).

[8] Borja Seijo-Pardo, Ver'onica Bol'on-Canedo, and Amparo Alonso-Betanzos. "Using a feature selection ensemble on DNA microarray datasets". In European Symposium on Artificial Neural Networks, Computational Intelligence and Deep learning, (2016).

[9] Luisa S. Brea, Noelia Barreira, Noelia S'anchez, Antonio Mosquera, Carlos Garc'ia-Res'ua, and Eva YebraPimentel. "On the analysis of feature selection techniques in a conjunctival hyperemia grading framework". In European Symposium on Artificial Neural Networks, Computational Intelligence and Deep learning,(2016).

[10] Luis Gon ${ }_{s}$ calves, Jorge Novo, and Aur'elio Campilho. "Feature definition, analysis and selection for lung nodule classification in chest computerized tomography images". In European Symposium on Artificial Neural Networks, Computational Intelligence and Deep learning, (2016).

[11] Soma Prathibha, Latha B, Suamthi G, "Particle swarm optimization based workflow scheduling for medical applications in cloud”, Biomedical Research”, (2017), Special Issue: S380-S385, pp.380-385.

[12] A Blog entitled Digital health, Virtual Hospital at A Conference on Virtual Clinical Trials under the website of Medically Home during May, 2019. [Last Accessed on 10-06-(2019)]

[13] Image collected from the Wikipedia address entitled "https://en.wikipedia.org/wiki/Proton_therapy". [Last Accessed on 10-06-(2019)] 